\title{
EXTENDED STRUCTURE OF Fe BIS AS COMPARED TO Fe $K$ EXAFS
}

\author{
E. Sobczak \\ Institute of Physics, Polish Academy of Sciences \\ Al. Lotników 32/46, 02-668 Warszawa, Poland
}

AND S. MOBILIO

INFN, Laboratori Nazionali di Frascati, 00044 Frascati, Italy

\begin{abstract}
The X-ray bremsstrahlung isochromat of Fe was measured for the photon energy $5415 \mathrm{eV}$ in an extended energy range up to $300 \mathrm{eV}$ above the threshold and compared to the Fe $K$-edge X-ray absorption spectrum. The Fourier analysis was performed for both spectra. It was stated that the extended structure of the Fe bremsstrahlung isochromat is clearly visible and is characteristic of the Fe bcc structure. Using the beat-node method, the distance between first and second shells in the bcc Fe was obtained quite close to the crystallographic value.
\end{abstract}

PACS numbers: 79.20.Kz, 78.70.Dm

Both X-ray bremsstrahlung isochromat spectra (BIS) and X-ray absorption spectra give information on the unoccupied electron states in the energy range above the Fermi level.

An initial electron state is quite different in BIS and X-ray absorption. It is a nearly-free-electron state in BIS and a core state with a well-defined orbital quantum number $l$ in the $\mathrm{X}$-ray absorption. Therefore, the selection rules for electron transitions are simple for X-ray absorption and rather complicated for BIS. The bremsstrahlung transitions are possible for all symmetry types of the final electron state, however, the probability of transition to the $p$-type electron states dominates over the others $[1,2]$. Therefore, BIS spectra are similar to the $K$-edge absorption spectra. The physical property of an X-ray absorption spectrum is a broadening due to the lifetime of a core hole, which does not exist in BIS.

Since 15 years, the extended X-ray absorption fine structure (EXAFS) is widely used for determination of the interatomic distances and nearest-neighbour configuration. Similar extended structures were observed in the X-ray bremsstrahlung isochromat spectra [2-5]. The extended X-ray bremsstrahlung isochromat fine structure (EXBIFS) of some fcc metals $\mathrm{Cu}, \mathrm{Ni}$, and Co was already compared with EXAFS at the $K$-edge of these metals [6]. Small differences between the EXBIFS 
and EXAFS oscillation phases occur due to different potential of the central atoms and contribution of the $s$ - and $d$-type final states in BIS.

In the present work the EXBIFS of Fe is studied and compared to EXAFS. BIS spectrum of Fe was measured at the photon energy $5415 \mathrm{eV}$. The instrumental details were described elsewhere [2]. The temperature of the Fe sample during the BIS experiment was about $800 \mathrm{~K}$. The $K$-edge absorption spectrum of $\mathrm{Fe}$ was measured at the liquid nitrogen temperature using the synchrotron radiation from the storage ring ADONE in Frascati.

The zero of the momentum scale was chosen in the middle of the small shoulder at the beginning of the threshold. The atomic contribution was found by fitting a polynomial of the third degree to each spectrum. The oscillation functions $\chi(k)$ for EXBIFS and EXAFS obtained by subtraction of atomic contributions are shown in Figs. 1a and 1b. The small amplitude of EXBIFS for big values of momentum $k$ is mainly caused by the high temperature of the BIS experiment.
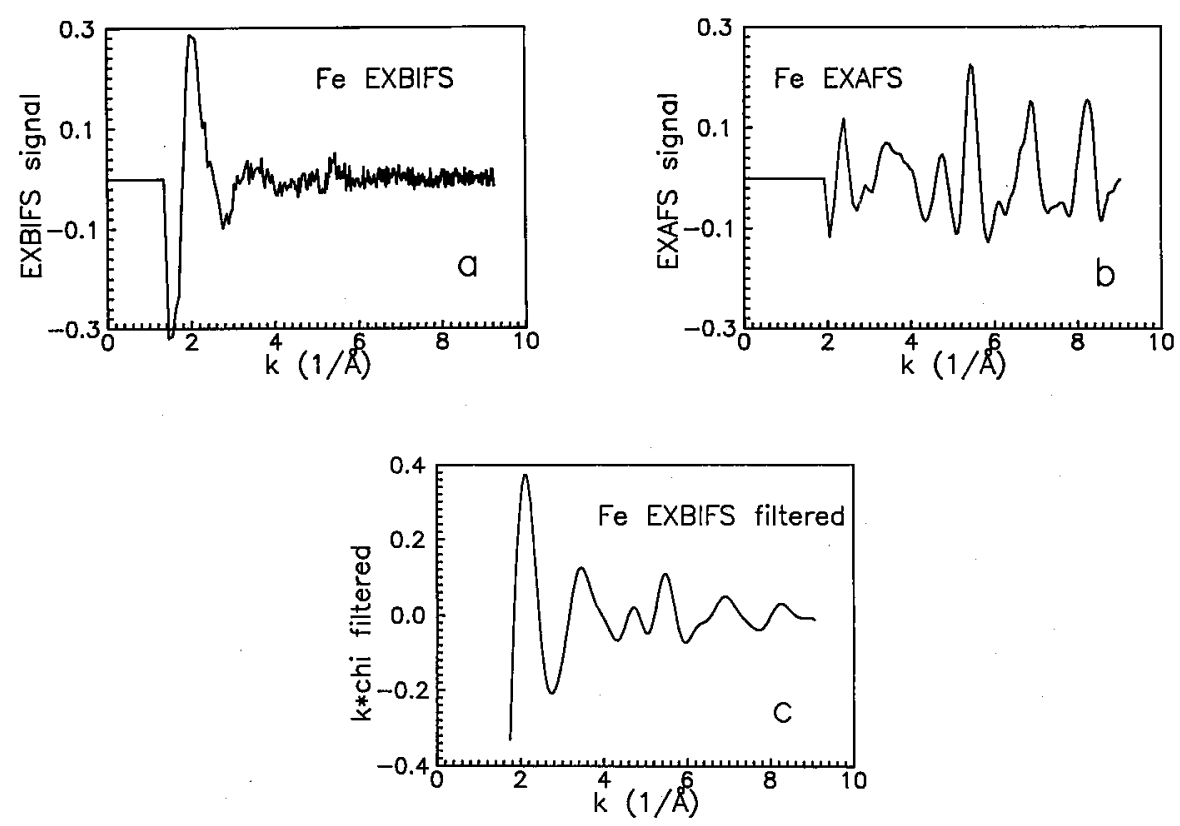

Fig. 1. Oscillation functions: (a) EXBIFS of Fe, (b) $K$-edge EXAFS of $\mathrm{Fe}$, (c) EXBIFS of Fe filtered in the range of 5 nearest shells.

The Fourier transforms (FT) of the EXBIFS and EXAFS signals were performed. The first- and second-shell FT peaks are not resolved because of the small intershell distance in the bcc structure equal to $0.384 \AA$ for the bcc Fe.

The noisy EXBIFS signal can be smoothed by the Fourier backtransform in the range of 5 nearest shells (from 1.5 to $5.0 \AA$ ). The result of such filtering scaled by $k$ is shown in Fig. 1c. The filtered EXBIFS is in good agreement with the $K$-edge EXAFS in Fig. $1 \mathrm{~b}$.

The Fourier backtransform of the first unresolved FT peak was calculated 

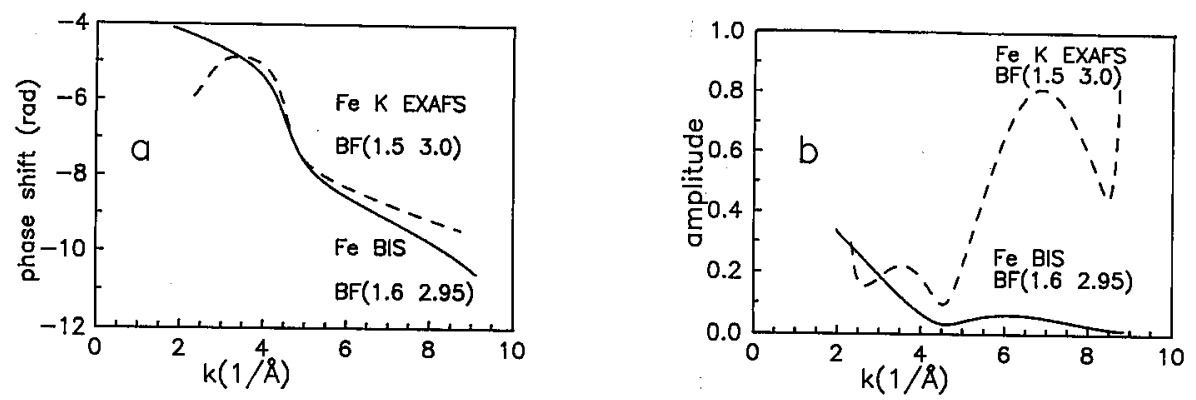

Fig. 2. The backtransforms for Fe EXBIFS (solid line) and $K$-edge EXAFS (dashed line): (a) phase shift, (b) amplitude.

in the range 1.6 to $2.95 \AA$ for EXBIFS and in the range 1.5 to $3.0 \AA$ for EXAFS. The phase shift of the backtransform shown in Fig. 2a shows a jump at the value $k$ being equal to 4.53 and $4.16 \AA^{-1}$ for EXBIFS and EXAFS, respectively. At the same points the amplitude of the backtransform in Fig. 2a shows a minimum. The beat-node method [7] was applied to evaluate the distance between first and second shells in the bcc Fe using the Fourier backtransform of the first unresolved FT peak. We obtained values of 0.38 and $0.35 \AA$ from EXAFS and EXBIFS respectively, which are quite close to the crystallographic value.

The main result of our work is that the extended structure of the Fe BIS spectrum is clearly visible and is characteristic of the bcc structure. Using the Fourier transform and beat-node method the first and second nearest-neighbours distances can be evaluated with accuracy of $0.05 \AA$ from the EXBIFS spectrum of Fe. The accuracy obtained in the EXBIFS analysis is lower than EXAFS due to the bigger statistical error, higher temperature and the $s$ - and $d$-symmetry contributions to BIS.

\section{References}

[1] A. Simůnek, J. Vackàr, E. Sobczak, Phys. Rev. B 38, 8515 (1988).

[2] E. Sobczak, J. Auleytner, Phys. Rev. B 37, 6251 (1988).

[3] E. Sobczak, R. Goldberg, J. Pelka, J. Auleytner, in: Inner-Shell and X-Ray Physics of Atoms and Solids, Eds. D.J. Fabian, H. Kleinpoppen, L.M. Watson, Plenum, New York 1981, p. 529.

[4] W. Speier, T.M. Hayes, J.W. Allen, J.B. Boyce, J.C. Fuggle, M. Campagna, Phys. Rev. Lett. 55, 1693 (1985).

[5] E. Sobczak, J. Auleytner, J. Phys. (France) 48, coll. C9, suppl. 12, 1121 (1987).

[6] E. Sobczak, J. Auleytner, S. Mobilio, A. Balerna, O. Smotlacha, Physica B 158, 553 (1989).

[7] G. Martens. P. Rabe, N. Schwentner, A. Werner, Phys. Rev. Lett. 9, 1411 (1977). 